\title{
The politics of development
}

\author{
Medical Innovations in Humanitarian \\ Situations: The Work of Médecins \\ Sans Frontières \\ Jean-Hervé Bradol and Claudine Vidal, \\ Editors \\ Médecins Sans Frontières; 2011
}

$\mathrm{T}$ he Chinese symbols for crisis mean both danger/peril and opportunity/crucial point. Médecins Sans Frontières (MSF) has illustrated both in this tightly edited, informative collection of essays, which is both well written and strong ly researched. It should be of interest to clinicians whether or not we work in crisis situations, as a concise history of the evolution of the treatment of selected infectious diseases, as well as the politics of development.

The book achieves its goal of giving us both a history of MSF and its role in medical innovation in crisis situations. It poses the question: "How can we create a momentum for change benefiting affected populations?" It takes a lessonslearned approach and challenges complacent policy-makers and politicians who cite "the poverty and ignorance of affected populations or (are) under the pretext of complying with international recommendations, economic constraints, and public authorities."

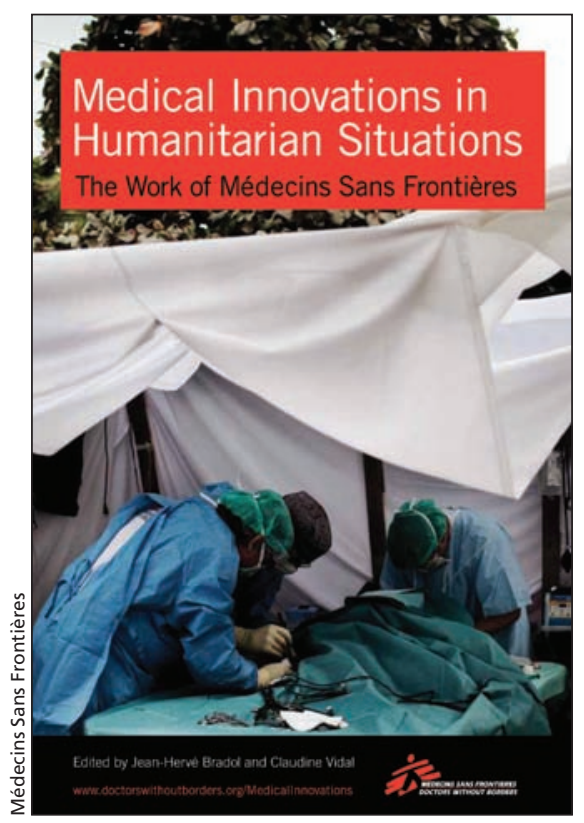

nal divisions within MSF are clearly discussed in this book, which confronts some of these debates - for example between MSF-France, MSF-International, and the Burundian government and the World Health Organization (WHO) over changing to artemisinincombination therapy in malaria treatment. Similarly in Niger, MSF-France took a confrontational position against the World Food Programme with respect to food supplementation using ready-to-

\section{MSF continues to be an inherently political organization.}

MSF has been an inherently political organization and this book openly discusses this aspect of its history. After breaking away from the neutral position of the Red Cross as a group of physicians responding to the Biafran conflict in 1967, MSF has continued to be a political and moral witness. Internally, the group has also had its own divisions with a parallel group forming under Bernard Kouchner in 1979. Inter- use therapeutic foods in cases of infant and child malnutrition, and highlighted the political dimensions of poverty, with which others within MSF disagreed.

Innovations are not simply new technologies, often they also create new dynamics: "Innovation upsets existing relationships between institutions, between practitioners, and between researchers. It produces tensions, shifting alliances, and public opinion cam- paigns." Field realities challenge the possibilities for randomized control trials. Scientific rigour is difficult in crisis situations. MSF has come into opposition, and then worked toward consensus, with United Nations agencies, researchers, drug manufacturers, development partners and host governments, in their passionate commitment to humanitarian action. Its role in the Campaign for Access to Essential Medicines leveraged MSF's reputation, contributing to its being awarded The Nobel Peace Prize in 1999.

MSF's contributions to improving the diagnosis and treatment of infectious diseases, including cholera, meningococcal meningitis, sleeping sickness, malaria and HIV, is impressive. For example, MSF helped to fieldtest simplified management protocols and used cholera treatment centres or camps to decompress hospitals.

In the meningococcal meningitis belt in sub-Saharan Africa, MSF helped optimize vaccination strategies and to fieldtest oily chloramphenicol (a long-acting, single dose intramuscular antibiotic) and ceftriaxone for treatment.

MSF has been treating patients with sleeping sickness (human African Trypanosomiasis), which has affected over 100000 people a year, since the 1980s, and now treats almost one-third of existing cases. It has had a profound influence in the shift from using arsenic for treatment, to eflornithine and eflornithine/nifurtimox combination therapy and upscaling the availability of the drugs by cofounding the Drugs for Neglected Diseases Initiative (DNDi) in public/private collaboration with pharmaceutical firms. This initiative was acknowledged by WHO.

MSF has also field-tested rapid diagnostic tests and lobbied for the shift to artemisinin-based combination therapy for malaria, which kills nearly one million people annually. 
Since 1989 MSF has worked in the fight against HIV, helping to field test antiretroviral drugs in prevention of parent-to-child transmission, working to simplify treatment regimes, and advocating politically for the improved availability of antiretroviral therapies at lower cost.

These innovations in crisis situations also require new ways of working. Logistics management and field-based epidemiology helped MSF to respond quickly, leading to satellite organizations "MSF-Logistique" and "Epicentre." Many MSF workers made simple but effective innovations - boxes made by local carpenters not only contained medicines and standard treatment guidelines, but could be used to aid in the examination of patients and the delivery of babies.

The history of crisis medicine is not only about successes. Important collective failures are noted, such as the tuberculosis epidemic, which is linked with the HIV pandemic. In MSF projects for $\mathrm{TB}$, one-half of patients are incorrectly diagnosed, the vaccine is relatively ineffective, the Directly Observed Treatment Short Course or DOTS has not controlled the disease, and antibiotic resistance is increasingly problematic.
These will be among the challenges for medical innovation for the future generation of humanitarian activists in crisis situations. The reflections in this book are helpful to prepare this next generation, by honestly addressing both successes and controversies and also recognizing the important interface between medicine and politics.

\section{Gretchen Roedde MD}

Family physician

International reproductive health care consultant

Temiskaming Shores, Ont.

CMAJ 2012. DOI:10.1503/cmaj.111732 